PROCEEDINGS OF THE

AMERICAN MATHEMATICAL SOCIETY

Volume 137, Number 8, August 2009, Pages 2631-2637

S 0002-9939(09)09869-4

Article electronically published on March 17, 2009

\title{
ON THE EIGENSPACES OF LAMPLIGHTER RANDOM WALKS AND PERCOLATION CLUSTERS ON GRAPHS
}

\author{
FRANZ LEHNER
}

(Communicated by Marius Junge)

\begin{abstract}
We show that the Plancherel measure of the lamplighter random walk on a graph coincides with the expected spectral measure of the absorbing random walk on the Bernoulli percolation clusters. In the subcritical regime the spectrum is pure point and we construct a complete orthonormal basis consisting of finitely supported eigenfunctions.
\end{abstract}

\section{INTRODUCTION}

The term lamplighter random walk has been introduced as a synonym for certain random walks on wreath products of groups. The spectrum of the lamplighter random walk on the infinite cyclic group attracted attention recently [2] when it was used to provide a counterexample to the so-called strong Atiyah conjecture.

In a previous paper [3] we studied the spectra of lamplighter random walks on arbitrary discrete groups, and we refer to that paper for a more detailed discussion. In the present paper we will study lamplighter random walks on arbitrary locally finite graphs and point out certain simplifications which emerge in a purely operator-theoretic treatment neglecting the group structure.

First we show by an elementary calculation the equivalence of the lamplighter random walk with the random walk on percolation clusters (Theorem 4.2) by comparison of moments, in the sense that the Plancherel measure of the lamplighter random walk coincides with the expected Plancherel measure of the absorbing random walk on the percolation cluster with parameter $\mathrm{p}=1 / m$, where $m$ is the number of different states of a lamp. The main result (Theorem 5.3) provides a spectral resolution of the transition operator which exhibits in more detail the intimate connection between lamplighter random walks and percolation clusters. In particular, in the subcritical case the transition operator has pure point spectrum and we construct a complete orthonormal basis of eigenfunctions, while in the supercritical phase the orthonormal system though still present ceases to be complete. It is still unknown whether a continuous spectrum occurs, and we leave this question for further investigation.

Received by the editors July 9, 2008.

2000 Mathematics Subject Classification. Primary 43A05, 47B80, 60K35.

Key words and phrases. Wreath product, percolation, random walk, spectral measure, point spectrum, eigenfunctions.

(C)2009 American Mathematical Society Reverts to public domain 28 years from publication 


\section{RANDOM WALKS ON GRAPHS}

Let $G=(V, E)$ be a connected graph and consider a stationary nearest neighbour random walk on $G$, i.e., a Markov chain $X_{n}$ with state space $V$ and transition probabilities

$$
\mathbf{P}\left[X_{n+1}=y \mid X_{n}=x\right]= \begin{cases}p(x, y) & \text { if } y \sim x \\ 0 & \text { otherwise }\end{cases}
$$

where $p(x, y)$ are fixed given probabilities. A lot of information about this Markov chain is contained in the transition operator

$$
T f(x)=\sum_{y \sim x} p(x, y) f(y)
$$

acting on the Hilbert space $\ell_{2}(G)=\left\{f: V \rightarrow \mathbf{C}: \sum_{x \in V}|f(x)|^{2}<\infty\right\}$ of square summable functions on (the vertices of) $G$. For example, let us record here the formula for the $n$-step return probability

$$
p^{(n)}(x, x)=\mathbf{P}\left[X_{n}=x \mid X_{0}=x\right]=\sum_{x_{1}, x_{2}, \ldots, x_{n-1}} p\left(x, x_{1}\right) p\left(x_{1}, x_{2}\right) \cdots p\left(x_{n-1}, x\right),
$$

which can also be written as

$$
p^{(n)}(x, x)=\left\langle T^{n} e_{x}, e_{x}\right\rangle,
$$

where $\left(e_{x}\right)_{x \in V}$ denotes the canonical basis of $l_{2}(G)$.

We will assume throughout that the transition operator is selfadjoint, and in this case the transition probabilities can be interpreted as the moments

$$
p^{(n)}(x, x)=\int t^{n} d \mu(t)
$$

of the Plancherel measure $d \mu(t)=\left\langle d E(t) e_{x}, e_{x}\right\rangle$, where $d E$ comes from the spectral resolution $T=\int t d E(t)$ of the operator $T$. This may serve as a motivation to study the spectrum of transition operators.

\section{LAMPLIGHTER RANDOM WALKS}

Let us define the lamplighter random walk on the graph $G$. We equip every vertex of $G$ with a lamp of $m$ colors or states, one of the colors being black, meaning that the lamp is turned off. Denote by $H$ the (finite) set of possible states of a lamp and by $m=|H|$ the number of different states. For practical purposes we will assume $H=\mathbf{Z}_{m}$, although the group structure of the latter will not be essential; we will use the convention that state 0 corresponds to a lamp being turned off. In addition to walking around, the random walker is now allowed to alter the states of the lamps in certain ways. This is encoded in a new state space.

Definition 3.1. A configuration on $G$ is a function $\eta: G \rightarrow H$ with finite support in the sense that the number of vertices $x$ with $\eta(x) \neq 0$ is finite; this reflects the fact that at any given time only finitely many lamps have been touched. We denote by $\mathfrak{C}=H^{(G)}$ the set of configurations.

The switch-walk-switch lamplighter random walk describes a random walker moving around in the graph according to the law (2.1). Before and after each step, he or she changes the state of the lamp in the current position at random, where the 
random steps and lamp operations are assumed to occur independently from each other. This way we obtain a Markov chain on the configuration space $\mathfrak{C} \times G$ with transition probabilities

$$
\tilde{p}(\xi, x ; \eta, y)=\frac{p(x, y)}{m^{2}}
$$

if $y \sim x$ and $\xi$ and $\eta$ coincide outside $x$ and $y$; otherwise, $\tilde{p}(\xi, x ; \eta, y)=0$. One can interpret the configuration space $\mathfrak{C} \times G$ again as a graph (the so-called lamplighter graph; see [1] for a generalization to so-called Diestel-Leader graphs) and using the neighbour relation on this graph the $n$-step return probability $\tilde{p}^{(n)}(\xi, x ; \xi, x)$ can be expressed by the corresponding expression (2.2). However, because of the assumption that each color is switched with the same probability, there is a simplification.

We start with the configuration $\iota$ where all lamps are off and the random walker is in some start vertex $x$. For each closed path $x_{0}, x_{1}, \ldots, x_{n}$ starting and ending in $x$ and a sequence of switching events, we are interested in the probability that after the $n$-th step all lamps are turned off again. Thus the intermediate states of the lamps are not important, and the only thing that matters is the final state of the lamps. However, the final state at each site only depends on the last visit, and therefore we have

$\mathbf{P}[$ at the end all lamps are off $]$

$$
=\mathbf{P}[\text { at the last visit each lamp is turned off }]=\left(\frac{1}{m}\right)^{\left|\left\{x_{0}, x_{1}, \ldots, x_{n}\right\}\right|},
$$

and for the $n$-step return probability we record the formula

$$
\tilde{p}^{(n)}(\iota, x ; \iota, x)=\sum_{x_{1}, x_{2}, \ldots, x_{n-1}} p\left(x, x_{1}\right) p\left(x_{1}, x_{2}\right) \cdots p\left(x_{n-1}, x\right) m^{-\left|\left\{x_{0}, x_{1}, \ldots, x_{n}\right\}\right|} .
$$

In the next section we shall compare this formula with another one which describes the return probabilities of random walks on percolation clusters.

\section{Bernoulli percolation}

Let $0<\mathrm{p}<1$. On the same graph $G$ as in the preceding section consider Bernoulli site percolation with parameter p; i.e., on the probability space $\Omega=$ $\{0,1\}^{G}$ we consider the i.i.d. random variables $\left(Y_{x}\right)_{x \in G}$ with Bernoulli distribution $\mathbf{P}\left[Y_{x}=1\right]=\mathrm{p}$. Given $\omega \in \Omega$, let $A(\omega)$ denote the subgraph of $G$ induced on $\left\{x: Y_{x}(\omega)=1\right\}$, and for any vertex $x \in G$, let $C_{x}(\omega)$ denote the connected component of $A(\omega)$ containing a vertex $x$, which is called the percolation cluster at $x$. It is well known that for every graph $G$ there is a critical parameter $\mathrm{p}_{c}$ such that for any vertex $x \in G$ a phase transition occurs in the sense that for $\mathrm{p}<\mathrm{p}_{c}$ the cluster $C_{x}$ is almost surely finite and for $\mathrm{p}>\mathrm{p}_{c}$ it is infinite with positive probability. In order to make use of this fact we recall a combinatorial interpretation of criticality.

Definition 4.1. For a subset $A \subseteq G$ we denote its vertex boundary by

$$
d A=\{y \in G: y \notin A, y \sim x \text { for some } x \in A\} .
$$

For $x \in G$, we denote by

$$
\mathcal{C}_{x}=\{A \subseteq G: x \in A, A \text { finite and connected }\}
$$

the set of finite path-connected neighbourhoods of $x$. In the case of $G=\mathbf{Z}^{2}$, these are sometimes called lattice animals. 
The probability of a fixed $A \in \mathcal{C}_{x}$ to occur as percolation cluster at $x$ is

$$
\mathbf{P}\left[C_{x}=A\right]=\mathrm{p}^{|A|}(1-\mathrm{p})^{|d A|}
$$

thus for $\mathrm{p}<\mathrm{p}_{c}$ we have

$$
\sum_{A \in \mathcal{C}_{x}} \mathrm{p}^{|A|}(1-\mathrm{p})^{|d A|}=1
$$

because some $A \in \mathcal{C}_{x}$ is reached almost surely.

Now consider the absorbing random walk on $C=C_{x}(\omega)$ :

$$
p_{C}(y, z)= \begin{cases}p(y, z) & \text { if } y, z \in C \\ 0 & \text { otherwise }\end{cases}
$$

and the random walker dies when a deleted vertex is reached. Our first observation is contained in the following generalization of [3, Theorem 1.1].

Theorem 4.2. If we set the percolation parameter $\mathrm{p}=1 / m$, we have

$$
\tilde{p}^{(n)}(\iota, x ; \iota, x)=\mathbf{E} p_{C}^{(n)}(x, x),
$$

and therefore the Plancherel measure of $\widetilde{T}$ coincides with the integrated density of states of the random walk on the percolation cluster $C_{x}$.

Proof. For a fixed cluster $C \in \mathcal{C}_{x}$ the $n$-step return probability of the absorbing random walk is

$$
\begin{aligned}
p_{C}^{(n)}(x, x) & =\sum_{x_{1}, x_{2}, \ldots, x_{n-1}} p_{C}\left(x, x_{1}\right) p_{C}\left(x_{1}, x_{2}\right) \cdots p_{C}\left(x_{n-1}, x\right) \\
& =\sum_{x_{1}, x_{2}, \ldots, x_{n-1}} p\left(x, x_{1}\right) p\left(x_{1}, x_{2}\right) \cdots p\left(x_{n-1}, x\right) \mathbf{1}_{\left[\left\{x, x_{1}, \ldots, x_{n-1}\right\} \subseteq C\right]} .
\end{aligned}
$$

Now taking the expectation of this return probability we get

$$
\mathbf{E} p_{C}^{(n)}(x, x)=\sum_{x_{1}, x_{2}, \ldots, x_{n-1}} p\left(x, x_{1}\right) p\left(x_{1}, x_{2}\right) \cdots p\left(x_{n-1}, x\right) \mathbf{p}^{\left|\left\{x, x_{1}, \ldots, x_{n-1}\right\}\right|}
$$

since

$$
\mathbf{E} \mathbf{1}_{\left[\left\{x, x_{1}, \ldots, x_{n-1}\right\} \subseteq C\right]}=\mathbf{P}\left[\left\{x, x_{1}, \ldots, x_{n-1}\right\} \subseteq C\right]=\mathrm{p}^{\left|\left\{x, x_{1}, \ldots, x_{n-1}\right\}\right|} .
$$

For $\mathrm{p}=1 / m$ this coincides with formula (3.1).

\section{Eigenfunctions}

In this section we construct eigenfunctions of the transition operator $\widetilde{T}$ of the lamplighter random walk. We identify $\mathcal{H}=l_{2}(\mathfrak{C} \times G)=l_{2}(\mathfrak{C}) \otimes_{2} l_{2}(G)$ with the space of square summable functions on $\mathfrak{C} \times G$. Denote the standard bases of $l_{2}(H)$ and $l_{2}(G)$ by $\left(e_{\eta}\right)_{\eta \in \mathfrak{C}}$ and $\left(e_{x}\right)_{x \in G}$, respectively, and the canonical basis elements of $\mathcal{H}$ by $e_{\eta, x}=e_{\eta} \otimes e_{x}$. For each $x \in G$ we need two projections: on the one hand the one-dimensional projections

$$
P_{x} e_{y}=\delta_{x y} e_{y}
$$

on $l_{2}(G)$, and on the other hand the averaging operators on $l_{2}(\mathfrak{C})$ given by

$$
\Theta_{x} f(\eta)=\frac{1}{m} \sum_{\eta^{\prime}} f\left(\eta^{\prime}\right)
$$


where $\eta^{\prime}$ runs over all $m$ different configurations which coincide with $\eta$ outside $x$. We will denote its amplification to $\mathcal{H}$ by $\widetilde{\Theta}_{x}=\Theta_{x} \otimes I$. Let us denote by $S_{x y}$ the partial isometry

$$
S_{x y} e_{z}=\delta_{y z} e_{x}
$$

on $l_{2}(G)$. Using this notation we can write the transition operator

$$
\widetilde{T} f(\eta, x)=\sum_{y \sim x} p(x, y) \widetilde{\Theta}_{x} \widetilde{\Theta}_{y} f(\eta, y)
$$

as

$$
\widetilde{T}=\sum_{x} \sum_{y \sim x} p(x, y) \Theta_{x} \Theta_{y} \otimes S_{y x},
$$

where the sum converges in the strong operator topology. Note that the operators $\widetilde{\Theta}_{x}$ commute with each other and with $S_{y z}$ and therefore they commute with $\widetilde{T}$.

Definition 5.1. For a finite subset $A \subseteq G$ with vertex boundary $d A$ we define the projection

$$
\Theta_{A, d A}=\prod_{x \in A} \Theta_{x} \prod_{y \in d A}\left(I-\Theta_{y}\right)
$$

and its amplification to $\mathcal{H}$ by $\widetilde{\Theta}_{A, d A}=\Theta_{A, d A} \otimes I$.

The following lemma collects the main properties of the projections $\Theta_{A, d A}$.

Lemma 5.2. (1) If $A$ and $B$ are connected subsets of $G$ with $A \cap B \neq \emptyset$, then $\Theta_{A, d A} \Theta_{B, d B}=0$.

(2) In the subcritical regime $\mathrm{p}<\mathrm{p}_{c}$ and for fixed $x \in G$, the family $\left(\Theta_{A, d A}\right)_{A \in \mathcal{C}_{x}}$ is a partition of unity on $l_{2}(\mathfrak{C})$; i.e., different projections are mutually orthogonal and their strong sum is $I$.

Proof. If the subgraphs $A \neq B$ are connected and contain a common vertex $x$, then one of them must intersect the vertex boundary of the other in some vertex $y$; thus $\Theta_{y}$ meets its complement $I-\Theta_{y}$ in the product $\Theta_{A, d A} \Theta_{B, d B}$ and they annihilate each other. Therefore $\Theta_{A, d A}$ and $\Theta_{B, d B}$ are orthogonal. To show completeness, consider the total set of vectors $\Omega=\left\{e_{\eta}: \eta \in \mathfrak{C}\right\}$ in the Hilbert space $l_{2}(\mathfrak{C})$. It spans a dense subspace and therefore it suffices to show that $\sum_{A \in \mathcal{C}_{x}} \Theta_{A, d A} e_{\eta}=e_{\eta}$ for each individual $\eta$. Since we have already shown that the projections $\left(\Theta_{A, d A} e_{\eta}\right)_{A \in \mathcal{C}_{x}}$ are mutually orthogonal, it suffices to show that

$$
\sum_{A \in \mathcal{C}_{x}}\left\|\Theta_{A, d A} e_{\eta}\right\|^{2}=1
$$

Now

$$
\left\|\Theta_{A, d A} e_{\eta, x}\right\|^{2}=\left\langle\Theta_{A, d A} e_{\eta, x}, e_{\eta, x}\right\rangle=\mathrm{p}^{|A|}(1-\mathrm{p})^{|d A|},
$$

and thus condition (5.1) is equivalent to condition (4.1), which is satisfied in the subcritical regime (and sometimes in the critical regime as well).

Theorem 5.3. In the subcritical regime, $\left(\Theta_{A, d A} \otimes P_{A}\right)_{A \subseteq G}$ finite, connected is a partition of unity on $\mathcal{H}$ and reduces $\widetilde{T}$ :

$$
\widetilde{T}\left(\Theta_{A, d A} \otimes P_{A}\right)=\Theta_{A, d A} \otimes T_{A},
$$

where

$$
T_{A}=P_{A} T P_{A}
$$


denotes the truncation of the transition operator $T$ of the simple random walk on $T$ to the subgraph $A$.

Proof. Indeed it follows from Lemma 5.2 that the family $\left(\Theta_{A, d A} \otimes P_{x}\right)_{x \in G, A \in \mathcal{C}_{x}}$ is a partition of unity of $l_{2}(\mathfrak{C} \times G)$ :

$$
\sum_{x \in G} \sum_{A \in \mathcal{C}_{x}} \Theta_{A, d A} \otimes P_{x}=I \otimes I,
$$

and interchanging the order of summation we obtain

$$
\sum_{A} \Theta_{A, d A} \otimes P_{A}=I \otimes I
$$

Now we show that each $\Theta_{A, d A} \otimes P_{A}$ reduces $\widetilde{T}$. First note that $\Theta_{A, d A}$ commutes with all $\Theta_{x}$ and therefore $\widetilde{\Theta}_{A, d A}$ commutes with $\widetilde{T}$. It suffices to show equality of the left- and right-hand sides of (5.2) evaluated at $f=e_{\eta, x}$ for every $\eta \in \mathfrak{C}$ and $x \in A$. If $x \notin A$, then both sides vanish and there is nothing to show. Assume now that $x \in A$. Then

$$
\begin{aligned}
\widetilde{T}\left(\Theta_{A} \otimes P_{A}\right) e_{\eta, x} & =\widetilde{\Theta}_{A, d A} \widetilde{T} e_{\eta, x} \\
& =\widetilde{\Theta}_{A, d A} \sum_{y \sim x} p(x, y) \widetilde{\Theta}_{x} \widetilde{\Theta}_{y} e_{\eta, y} \\
& =\widetilde{\Theta}_{A, d A} \sum_{\substack{y \sim x \\
y \in A}} p(x, y) e_{\eta, y} \\
& =\left(\Theta_{A, d A} \otimes T_{A}\right) e_{\eta, x} .
\end{aligned}
$$

Corollary 5.4. In the subcritical regime there exists a complete orthonormal system of finitely supported eigenfunctions of $\widetilde{T}$.

Proof. If we denote for each finite connected subgraph $A \subseteq G$ by $\left\{f_{a}: a \in A\right\}$ a basis of $l_{2}(A) \subseteq l_{2}(G)$ consisting of eigenfunctions of $T_{A}$, then the eigenspaces of $\widetilde{T}$ are given by $\left\{\Theta_{A, d A} l_{2}(\mathfrak{C}) \otimes f_{a}: A \subseteq G\right.$ connected, $\left.a \in A\right\}$. For every finite connected subset $A \subseteq G$ we apply the Gram-Schmidt procedure to $\left\{\Theta_{A, d A} e_{\eta}: \eta \in \mathfrak{C}\right\}$ to obtain a basis of $\Theta_{A, d A} l_{2}(\mathfrak{C})$ consisting of finitely supported functions $\left(\varphi_{A, i}\right)_{i \in \mathbf{N}}$. This is possible because $\Theta_{A, d A} e_{\eta}$ has finite support for each $\eta \in \mathfrak{C}$ and $l_{2}(\mathfrak{C})$ is separable. Putting these functions together with the eigenfunctions $f_{a}$ we obtain the eigenbasis

$$
\left\{\varphi_{A, i} \otimes f_{a}: A \subseteq G, i \in \mathbf{N}, a \in A\right\} .
$$

\section{Concluding Remarks}

(1) In the case where the graph $G$ is a Cayley graph as considered in [3], the projections $\Theta_{A, d A} \otimes P_{A}$ are not necessarily elements of the group algebra, and therefore the partition of unity obtained in the present paper is different from the one obtained in [3].

(2) Similar results hold if the lamps are placed on the edges; see [3] for a discussion. The preceding considerations also hold when one allows the number of colors (and accordingly the percolation parameter) to vary among the 
vertices $x$; however, for the sake of simplicity only identical lamps on all vertices were considered here.

(3) It is not essential that the projections $\Theta_{x}$ are averaging operators and $\mathrm{p}=$ $1 / m$. As discussed in [3], similar deterministic models can be constructed for arbitrary percolation parameters $\mathrm{p}$. One has to replace the matrix algebra operating on the colour space $l_{2}(H)$ by an infinite-dimensional finite von Neumann algebra and take a projection $\Theta$ of trace $p$ from that algebra. We leave the details to the reader.

(4) It is still unknown what happens in the supercritical regime, where it is conjectured that a continuous spectrum occurs at least in some cases. It may be hoped that the new approach will lead to some insight into this question.

\section{REFERENCES}

1. Laurent Bartholdi and Wolfgang Woess, Spectral computations on lamplighter groups and Diestel-Leader graphs, J. Fourier Anal. Appl. 11 (2005), no. 2, 175-202. MR2131635 (2006e:20052)

2. Rostislav I. Grigorchuk, Peter Linnell, Thomas Schick, and Andrzej Żuk, On a question of Atiyah, C. R. Acad. Sci. Paris Sér. I Math. 331 (2000), no. 9, 663-668. MR.1797748 (2001m:57050)

3. Franz Lehner, Markus Neuhauser, and Wolfgang Woess, On the spectrum of lamplighter groups and percolation clusters, Math. Ann. 342 (2008), no. 1, 69-89. MR2415315

Institut für Mathematische Strukturtheorie, Steyrergasse 30, A-8010 Graz, Austria

E-mail address: lehner@finanz.math.tu-graz.ac.at 